Abanico Veterinario. Enero-Diciembre 2021; 11:1-14. http://dx.doi.org/10.21929/abavet2021.3

Artículo Original. Recibido: 03/09/2020. Aceptado: 21/12/2020. Publicado: 17/01/2021. Clave:2020-38.

\title{
Evaluación de extractos vegetales para el control de Oesophagostomun dentatum en cerdos pelón mexicano
}

\author{
Plant extracts evaluation for the control of Oesophagostomum dentatum in Mexican \\ Pelon pigs
}
García-Munguía Carlos ${ }^{11 D}$, Puentes Carolina1 ID, García-Munguía Alberto*2 ID, Haubi- Segura Carlos² ID, Sánchez-Chiprés David ${ }^{3}$ ID, García-Munguía Otilio 4 ID

${ }^{1}$ Departamento de Veterinaria y Zootecnia, Universidad de Guanajuato. Carretera Irapuato-Silao km 9, CP 36500 Irapuato, Guanajuato, México. ${ }^{2}$ Centro de Ciencias Agropecuarias, Universidad Autónoma de Aguascalientes. Av Universidad 940, col. Ciudad Universitaria, CP 20131, Aguascalientes, Aguascalientes. México. ${ }^{3}$ Centro Universitario de Ciencias Biológicas y Agropecuarias, Universidad de Guadalajara. Camino Ramón Padilla Sánchez 2100 Nextipac, 45200 Zapopan, Jalisco, México, ${ }^{4}$ Centro de Ciencias Económico Administrativas, Av Universidad 940, col. Ciudad Universitaria, CP 20131, Aguascalientes, Aguascalientes. México. *Autor responsable: García-Munguía Carlos, *Autor para correspondencia: García-Munguía Alberto, correo electrónico. almagamu@hotmail.com. cagamu@hotmail.com, puentecaro@hotmail.com, almagamu@hotmail.com,dhaubi@yahoo.com.chipres9@hotmail.com, einsteinoti@hotmail.com

\section{RESUMEN}

En México, el Oesophagostomum dentatum es considerado uno de los principales endoparásitos gastrointestinales que afecta a la raza de mayor importancia en la porcicultura rural [Cerdo Pelón Mexicano (CPM)]. En esta investigación se evaluó la eficacia biológica in vitro de extractos vegetales de jengibre, hierbabuena, tomillo y orégano, con el objetivo de encontrar nuevas alternativas de carácter natural para el control de Oesophagostomum dentatum; siendo comparada su eficiencia con ivermectina (IVM) y dimetilsulfóxido (DMSO). Durante la investigación, se colectaron 380 muestras de excretas (extraídas directamente del ano) de CPM, con un peso promedio ( $\pm D E) 40 \pm 5 \mathrm{~kg}$ por animal. Dichas muestras fueron analizadas mediante la técnica de McMaster, logrando identificar huevos de Oesophagostomum dentatum. La evaluación de la eficacia de los tratamientos se realizó en microplacas de cultivo celular incubadas durante $48 \mathrm{~h}$ a $25 \pm 1^{\circ} \mathrm{C}$ utilizando diferentes dosis de los extractos vegetales y comparando su eficacia de control con IVM y DMSO. Obteniendo que la efectividad biológica del extracto de jengibre (3\%) es similar al de la IVM (1\%), logrando la eliminación e inmovilización de la larva de Oesophagostomum dentatum en un $62 \%$. Mientras que los extractos de orégano, tomillo y hierbabuena presentaron un porcentaje de efectividad biológica menor al $20 \%$.

Palabras claves: parásitos, nematodos, gastrointestinales, Sus scrofas domesticus, jengibre.

\begin{abstract}
In Mexico, the Oesophagostomum dentatum is considered one of the main gastrointestinal endoparasites affecting the most important breed in rural pig farming [Cerdo Pelon Mexicano (CPM)]. In this research, it was evaluated the in vitro biological efficacy of vegetable extracts of ginger, mint, thyme and oregano, with the aim of finding new natural alternatives for the control of Oesophagostomum dentatum; being compared its efficiency with ivermectin (IVM) and dimethylsulfoxide (DMSO). During the investigation, 380 samples of excrements (extracted directly from the anus) of CPM were collected, with an average weight $( \pm D E) 40 \pm 5$ $\mathrm{kg}$ per animal. These samples were analyzed by means of McMaster technique, achieving to identify Oesophagostomum dentatum eggs. The evaluation of the treatments' effectiveness was carried out in cell culture microplates incubated for $48 \mathrm{~h}$ at $25 \pm 1 \stackrel{\circ}{\circ} \mathrm{C}$ using different doses of the vegetable extracts and
\end{abstract}


comparing their control effectiveness with IVM and DMSO. Obtaining that the biological effectiveness of the ginger extract $(3 \%)$ is similar to that of the IVM (1\%), achieving the elimination and immobilization of the Oesophagostomum dentatum larva in $62 \%$. While the extracts of oregano, thyme and mint presented a percentage of biological effectiveness of less than $20 \%$.

Keywords: parasites, gastrointestinal, nematodes, Sus scrofas domesticus, ginger.

\section{INTRODUCCIÓN}

Durante los últimos años, en México, la producción porcícola ha generado más de 350,000 empleos directos y 1.7 millones de empleos indirectos, provocando un crecimiento exponencial del $10.79 \%$, consecuencia de los aumentos de producción y una mejoría de precios en el mercado para el consumo de esta carne (Rebollar et al., 2016). Una de las actividades más importantes dentro del país es la porcinocultura rural, siendo el cerdo Pelón Mexicano (CPM) uno de sus grandes protagonistas, pues éste ha sido caracterizado principalmente por su rusticidad y variada alimentación (Lemus y Ly, 2010).Sin embargo, este tipo de producción se ve afectada por la presencia de parásitos que limitan el potencial productivo de los cerdos, provocando la pérdida del apetito y respuesta inmunológica; consecuentemente a ello una disminución en los pesos vivos y alteraciones en los índices de conversión alimenticia (Louie et al., 2007).

Es importante resaltar que la prevalencia de la parasitosis depende exclusivamente del sistema de manejo, de las condiciones de sanidad e higiene y de diferentes tipos de variables, como el clima, temperatura y humedad, que influyen en los ciclos de vida de los parásitos (Frontera et al., 2009); donde uno de los parásitos con mayor prevalencia en la producción porcícola es Oesophagostomum dentatum (Cordero et al., 2000). Actualmente los métodos de control que se han optado para este tipo de parasitosis han sido cada vez menos efectivos, debido a que estos nematodos han tenido una rápida evolución y desarrollo de resistencia contra los productos químicos utilizados para su control, lo que representa un riesgo para la salud humana (Taylor et al., 2009a). En la actualidad tres grandes familias de antiparasitarios son usados frecuentemente por los porcicultores, las lactonas macrocíclicas (IVM, moxidectina, doramectina), imidazoles tetrahidropirimidina (levamisol, moratel) y benzimidazoles (fenbendazol, oxfendazol y albendazol), dependiendo de las áreas en las que se desarrolla la producción (Encalada et al., 2014). El abuso de estos productos químicos ha ocasionado un problema de resistencia a los antiparasitarios (Kaplan y Vidyashankar, 2012); además, su mal uso puede ocasionar que estos lleguen al medio ambiente como un compuesto igual (sin cambios) o como un metabolito; para después transportarse y distribuirse en el agua, sedimentos, suelo y la flora (Horvat et al., 2012), causando alteraciones considerables en el ecosistema.

Por esta razón existe un creciente interés en explorar alternativas naturales, con propiedades capaces de actuar como bacteriostáticos, bactericidas y antiparasitarias (Aguilera, 2012). Las plantas, como parte de su metabolismo, sintetizan distintos componentes llamados metabolitos secundarios (Dávila et al., 2017). Diversas 
investigaciones realizadas han mostrado una gran diversidad de plantas que poseen estos metabolitos capaces de inhibir el crecimiento y desarrollo de patógenos (Rizo et al., 2017).

El objetivo del presente estudio fue evaluar la eficacia biológica de distintos extractos vegetales, como: jengibre (Zingiber officinale), orégano (Origanum vulgare), tomillo (Thymus) y hierbabuena (Mentha spicata); reportados anteriormente por sus compuestos activos capaces de actuar como bactericidas; comparándolos con productos comerciales que se utilizan actualmente para el control de Oesophagostomum dentatum presentes en CPM.

\section{MATERIAL Y MÉTODOS}

Zona de estudio: el estudio se desarrolló en el Centro de Conservación del Cerdo Pelón Mexicano y en el Laboratorio de Parasitología y Control Biológico, de la División de Ciencias de la Vida de la Universidad de Guanajuato. Las muestras fueron recolectadas de CPM, de pesos promedios $40 \pm 5 \mathrm{~kg}$, originarios de zonas rurales del municipio de Huehuetla, Hidalgo y Zacapoaxtla, Puebla, México. Se recolectaron un total de 380 muestras tomadas del recto y fueron colocadas en bolsas de polietileno debidamente identificadas (Aguilar et al, 2016); siendo éstas trasladadas al laboratorio, donde fueron almacenadas a una temperatura de $4 \pm 1^{\circ} \mathrm{C}$ hasta su procesamiento, el cual no fue mayor a $48 \mathrm{~h}$.

Material vegetativo: se colectaron hojas frescas de hierbabuena (Mentha spicata), tomillo (Thymus) y orégano (Origanum vulgare) (1 kg aproximadamente por muestra) en el municipio de Zacapoaxtla, Puebla, localizado a una altitud de 1825 m. s.n. m., así como bulbos de jengibre (Zingiber officinale) ( $2 \mathrm{~kg}$ aproximadamente) en el municipio de Huehuetla, Hidalgo, localizado a una altitud de 520 m. s. n. m. El material se almacenó y trasladó en refrigeración a $4^{\circ} \mathrm{C}$ en un mini refrigerador portátil (Chefman / RJ48-BLACK; Cooling \& Heating Company, United States), para evitar cambios en su composición (Salem et al., 2006). Posteriormente se sometieron a un proceso de secado bajo la sombra durante una semana, y finalmente tanto las hojas y bulbos se trituraron en un molino semi-industrial a un tamaño de $1 \mathrm{~mm}$ aproximadamente.

Obtención del extracto hidro-alcohólico (HA): por cada muestra se utilizaron $100 \mathrm{~g}$ y se sometieron a un proceso de maceración con una mezcla de agua y metanol (70:30 $\mathrm{v} / \mathrm{v}$ ) durante $24 \mathrm{~h}$, posteriormente se filtró la solución mediante diferentes filtros, utilizando (gasa y papel filtro) para obtener un extracto libre de impurezas. Una vez obtenido el extracto, se congeló a $-42{ }^{\circ} \mathrm{C}$ y finalmente se realizó el proceso de liofilización (liofilizador 7670520; LABCONCO, Kansas City, United States). El extracto liofilizado fue congelado para su posterior uso (Salem et al., 2006).

Material biológico: se realizó el diagnóstico parasitológico mediante la técnica de almacenamiento anaeróbico de huevos, descripta por (Coles et al., 2006) modificada; que consiste en procesar las muestras mediante la técnica de sedimentación que se 
encuentra en el contenido fecal y permitir que los huevos de parásitos se concentren en el fondo del tubo falcón; siendo así que en cada tubo falcón se colocaron $30 \mathrm{~mL}$ de agua y $4 \mathrm{~g}$ de heces, se homogenizó la muestra, vertiéndola en un tamiz y centrifugó por 5 min a $300 \mathrm{rpm}$. Posteriormente se realizó la técnica de flotación con $30 \mathrm{~mL}$ de solución glucosada con densidad de 1:200 (Cringoli et al., 2004); logrando de esta manera que los huevos de parásitos del fondo del tubo falcón floten por consecuencia de la densidad de la solución; nuevamente se centrifugó la mezcla por $5 \mathrm{~min}$ a $300 \mathrm{rpm}$. Finalmente se realizó nuevamente la técnica de sedimentación con $30 \mathrm{mLde}$ agua destilada para concentrar los huevos en el fondo del tubo centrifugando a $300 \mathrm{rpm}$ y establecer la concentración de huevos por $\mathrm{mL}$ de agua destilada esterilizada.

Técnica de McMaster: se realizó la técnica coproparasitoscópica de McMaster, utilizando una solución saturada de Sheather con una gravedad específica de 1.200 para estimar la cantidad de huevos por $\mathrm{g}$ de excremento, siendo la gravedad apropiada para llevar a cabo la técnica (Cringoli et al., 2004). Se utilizaron $2 \mathrm{~g}$ de excremento y $28 \mathrm{~mL}$ de solución saturada; la muestra se homogenizó y se colocó en la cámara de McMaster (MM-OP; PROLAB, Jalisco, México), utilizando una pipeta con una gasa como filtro, evitando la obstrucción en la revisión de la muestra. Se colocó el volumen necesario para realizar la lectura de un lado de la cámara, dejando reposar 2 min antes de ser observada al microscopio y llevar a cabo el conteo de huevos (Rodríguez et al., 2016).

Para la estimación del número de huevos por $\mathrm{g}$ de excremento se ajustó con el volumen total obtenido de mezclar el excremento y la solución de $30 \mathrm{~mL}$; considerando que cada compartimiento de la cámara mide $1 \mathrm{~cm}^{2}$ con una altura de $0.15 \mathrm{~cm}$, por lo que la lectura de ambos compartimientos es de $0.30 \mathrm{~mL}$ del volumen inicial total de $30 \mathrm{~mL}$ (Rodríguez et al., 2016). Se obtuvo la concentración de huevos por $\mathrm{g}$ de heces del parásito encontrados por medio de la fórmula de McMaster (Bowman, 2013).

$\operatorname{Total}\left(\frac{n^{\circ} \text { de huevos }}{g \text { de heces }}\right)=\frac{\text { huevos contados } x\left(\frac{\text { Vol.total }}{\text { Vol.celdas }}\right)}{g \text { de heces }}$

Los huevos de parásito fueron identificados con las claves morfológicas (Coffin, 1952), y el diagnóstico larvario se realizó con las claves morfológicas de Van Wyk et al., (2004), pudiéndose observar la extremidad craneal de las larvas, los apéndices terminales y la morfología de la cola.

Diseño experimental: el experimento se estableció bajo un diseño completamente al azar de 19 tratamientos de 20 repeticiones cada uno, teniendo en total de 380 unidades experimentales; cada unidad experimental constó de $1200 \mu \mathrm{L}(1100 \mu \mathrm{L}$ de extracto de jengibre más $100 \mu \mathrm{L}$ de solución con 70 larvas de nematodos). Cada unidad experimental estuvo conformada por un total de $100 \mu \mathrm{L}$ de nematodos vivos (con un promedio de 70 larvas). 
Prueba de inhibición de la migración larvaria: se llevó a cabo un cultivo larvario para llevar a los nematodos a la tercera etapa de la larva; para ello se les brindó las condiciones necesarias para la eclosión del huevo, realizando modificaciones a conveniencia a la técnica de McArthur et al., (2015). Se colocó excremento en recipientes de plástico perforados, con el objetivo de brindar un ambiente aeróbico, se adicionó aserrín estéril y se homogenizó el excremento adicionando agua destilada estéril. Se encubó la mezcla a 23-25 ㄷ durante 10 d, con una evaluación visual diaria en caso de requerir adición de humedad y oxigenar las muestras, removiendo el cultivo con ayuda de una espátula. Una vez concluido el tiempo de incubación, se colocó el cultivo en un embudo de Baermann; de esta manera se separaron las larvas de Oesphagostomum dentatum del contenido fecal. Tras un día en el embudo se obtuvo el líquido y mediante centrifugación se concentraron las larvas, para posteriormente realizar su conteo y diluciones, siendo identificadas con las claves morfológicas descriptas por Quiroz (2011). Dosis y número de aplicaciones: se realizaron evaluaciones con dosis de extracto de jengibre (Zingiber officinale) al 0.1\%, 0.3\%, 0.5\%, 0.7\%, 0.9\%, 1\%, 3\% (Gawel et al., 2003); orégano (Origanum vulgare) al $1 \%$, 3\% y 5\%, según lo mencionado por Borbolla y Velásquez, 2004 (citado en Guerra et al., 2008); tomillo (Thymus vulgaris) al 1\%, 3\% y 5\% (Ramos y Hernández, 2018); hierbabuena (Mentha spicata) al 1\%, 3\% y 5\% (Lagarto et al, 1997); comparando el efecto de cada uno con el testigo de agua, DMSO 5\% (Rendal et al., 2004) e IVM al 1\% (Chávez et al., 2006).

Para la dilución de los nematodos se utilizó una pipeta graduada en $100 \mu \mathrm{L}$, con la cual se extrajo la muestra recogida del embudo, depositados en cada pozo de la microplaca celular de $1200 \mu \mathrm{L}$ de volumen, una muestra de $100 \mu \mathrm{L}$ de nematodos; repitiendo el mismo procedimiento para cada unidad experimental. Para las unidades experimentales de los extractos de los tratamientos orgánicos a evaluar, se utilizó una solución madre del extracto al $5 \%$ (equivalente a $2 \mathrm{~mL}$ de extracto $+38 \mathrm{~mL}$ de agua destilada $=40 \mathrm{~mL}$ de solución); calculando así los equivalentes a cada porcentaje como se mencionaron anteriormente para cada unidad experimental según su porcentaje de inclusión.

Para la dilución de cada dosis de tratamiento se depositó la cantidad necesaria de agua destilada hasta completar los $1200 \mu \mathrm{L}$ de volumen, en cada unidad experimental. Al tratamiento Testigo se le adicionó con $100 \mu \mathrm{L}$ de nematodos y un total de $1100 \mu \mathrm{L}$ de agua destilada.

Durante el experimento se realizó una sola aplicación de los antiparasitarios, y con ayuda de un contador manual se realizó el conteo de los nematodos vivos y muertos de cada unidad experimental, a través de un microscopio con el objetivo 10x y 40x.

Análisis estadístico: para la evaluación de cada antiparasitario se determinó su efectividad, comparándola con el grupo control (Barrere et al., 2013). Los porcentajes de mortalidad se ajustaron con la fórmula de Abbott (Abbott,1987), y se realizó un análisis de varianza de los diferentes tratamientos y se hizo una comparación de medias mediante una prueba de Tukey al 95\% de confianza, con el paquete estadístico Statgraphics 9.0 (Cosialls et al., 2000). 


\section{RESULTADOS Y DISCUSIÓN}

El porcentaje de efectividad biológica de los diferentes antiparasitarios evaluados, fue el siguiente: primera evaluación $(0 \mathrm{~h})$, fue de $0 \%$ en cada tratamiento; esto por ser el primer conteo después de la aplicación. Para la segunda evaluación ( $24 \mathrm{~h}$ después de la aplicación de los tratamientos), no se mostraron diferencias significativas en la mortalidad de nematodos entre los tratamientos evaluados. En la tercera evaluación (48 h después de la aplicación de los tratamientos), se observó que el porcentaje de efectividad biológica de los diferentes tratamientos aumentó; sin embargo la IVM (1\%) y el extracto de jengibre (Zingiber officinale) (3\%) fueron los que presentaron mayor efectividad para el control de Oesophagostomum dentatum (cuadro 1).

\section{Cuadro 1. Porcentaje de Efectividad Biológica de los desparasitantes y extractos vegetales} evaluados

\begin{tabular}{|c|c|c|c|c|}
\hline Desparasitante & \% Inclusión & $\begin{array}{c}\text { Evaluación } 1 \\
(0 \text { horas })\end{array}$ & $\begin{array}{l}\text { Evaluación } 2 \\
\text { (24 horas) }\end{array}$ & $\begin{array}{c}\text { Evaluación } 3 \\
\text { (48 horas) }\end{array}$ \\
\hline \multicolumn{5}{|c|}{$\%$} \\
\hline $\mathrm{T}_{1}$ : Testigo $\mathrm{H}_{2} \mathrm{O}$ & --- & 0 & 0 & 0 \\
\hline $\mathrm{T}_{2}$ : Ivermectina & 1 & 0 & 32.56 & 62.72 \\
\hline $\mathrm{T}_{3}$ : DMSO & 5 & 0 & 0 & 10.02 \\
\hline $\mathrm{T}_{4}$ : Jengibre & 0.1 & 0 & 1.19 & 2.08 \\
\hline$T_{5}$ : Jengibre & 0.3 & 0 & 2.37 & 5.19 \\
\hline $\mathrm{T}_{6}$ : Jengibre & 0.5 & 0 & 6.15 & 11.46 \\
\hline $\mathrm{T}_{7}$ : Jengibre & 0.7 & 0 & 12.74 & 24.27 \\
\hline$T_{8}$ : Jengibre & 0.9 & 0 & 19.54 & 36.09 \\
\hline$T_{9}$ : Jengibre & 1 & 0 & 22.69 & 45.36 \\
\hline$T_{10}$ : Jengibre & 3 & 0 & 32.54 & 62.93 \\
\hline $\mathrm{T}_{11}$ : Hierbabuena & 1 & 0 & 1.67 & 3.04 \\
\hline $\mathrm{T}_{12}$ : Hierbabuena & 3 & 0 & 2.54 & 5.98 \\
\hline $\mathrm{T}_{13}$ : Hierbabuena & 5 & 0 & 4.66 & 9.53 \\
\hline $\mathrm{T}_{14}$ : Tomillo & 1 & 0 & 1.49 & 2.49 \\
\hline$T_{15}$ : Tomillo & 3 & 0 & 2.71 & 5.80 \\
\hline$T_{16}:$ Tomillo & 5 & 0 & 3.85 & 8.03 \\
\hline $\mathrm{T}_{17}$ : Orégano & 1 & 0 & 3.29 & 6.48 \\
\hline $\mathrm{T}_{18}$ : Orégano & 3 & 0 & 5.48 & 11.18 \\
\hline$T_{19}$ : Orégano & 5 & 0 & 8.80 & 17.02 \\
\hline
\end{tabular}

En la figura 1 se observa que la efectividad biológica del extracto de jengibre (Zingiber officinale) $(3 \%)$ es similar al de la IVM (1\%), en un 62\%; teniendo este extracto un crecimiento exponencial del $26.76 \%$ en promedio al ir aumentando su porcentaje de concentración; mientras que los extractos de orégano (Origanum vulgare), tomillo (Thymus) y hierbabuena (Mentha spicata), mantienen un porcentaje de efectividad biológica menor al 20\%; siendo estos poco eficaces en la mortalidad del nematodo evaluado. Según lo reportado por un estudio de Taylor et al. (2009a), se ha comprobado una resistencia múltiple de los nematodos gastrointestinales a las familias principales de antiparasitarios (benimidazoles, imidazoles y lactosas microcíclicas). Geurden et al. 
(2015), reportaron que la IVM ha sido uno de los desparasitantes más utilizados en los últimos 40 años, debido a la resistencia que los parásitos desarrollan en diferentes sitios de Alemania, Francia, Inglaterra e Italia. Se estudiaron 40 Unidades de Producción Animal (753 animales), llevando un registro de los huevos observados en el excremento y observando una disminución de la efectividad de la IVM y la moxidectina en las 8 Unidades de Producción Animal.

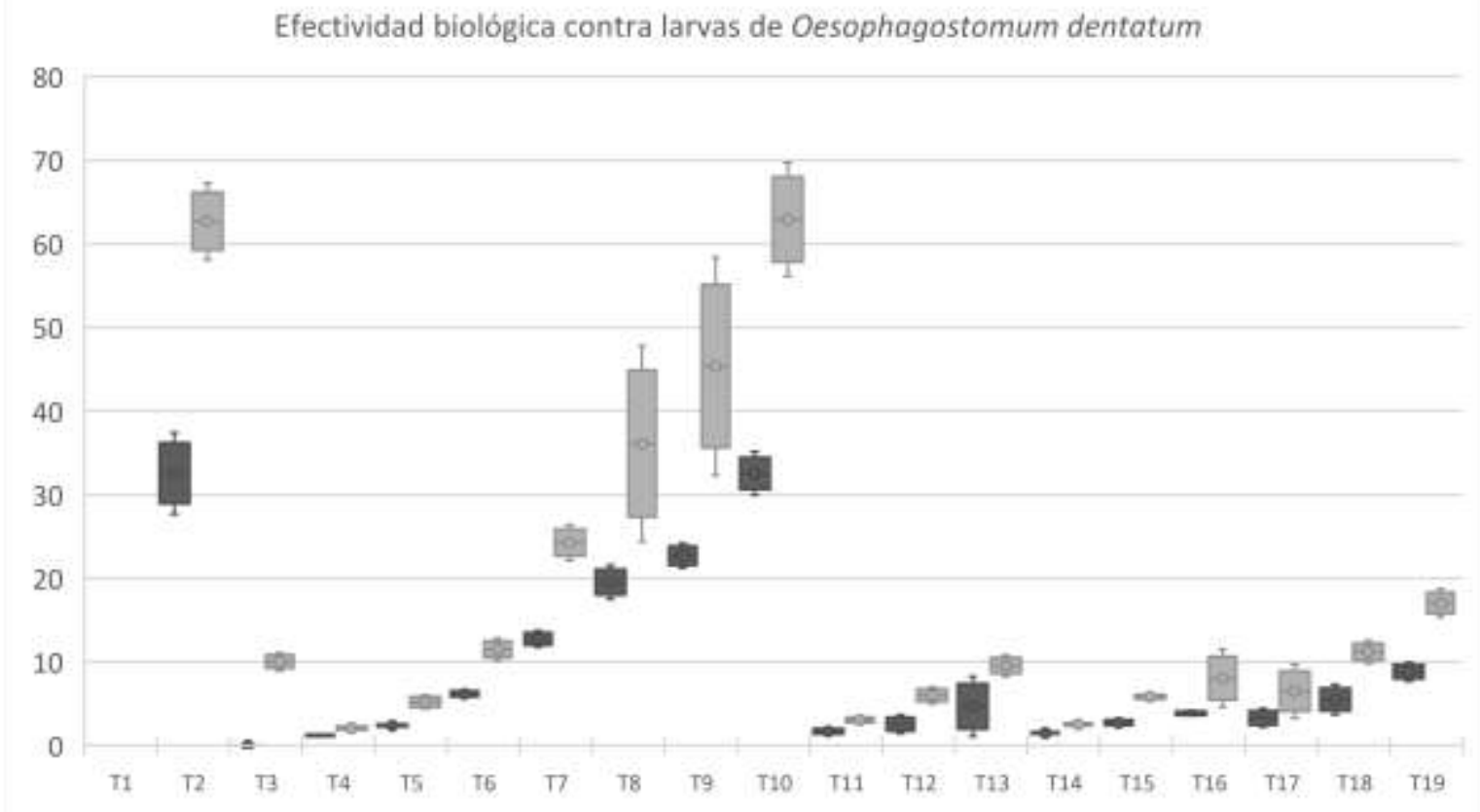

Eval 1 (24 hrs, dda) Eval 2 ( 48 hrs. dda)

dda: después de la aplicación

Figura 1. Porcentaje de Efectividad Biológica de cada desparasitante utilizado sobre las larvas de Oesophagostomum dentatum durante la evaluación 1 (a las 24 h después de aplicado el tratamiento) y en la evaluación 2 (a las 48 h después de aplicado el tratamiento)

En México, Alonso et al., (2015) evaluaron en 21 Unidades de Producción Animal en el estado de Veracruz, durante el periodo de enero 2012- abril 2013; entre las cuales únicamente en 2 Unidades de Producción Animal tienen parásitos susceptibles a la IVM, siendo las otras 15 las que presentan resistencia; además lograron identificar mediante cuestionarios que este problema se origina principalmente porque se realiza una práctica de desparasitación inadecuada; siendo uno de los principales problemas en el estado de Guanajuato, ya que los productores indican desparasitar sin un control adecuado, lo que generó que únicamente 2 Unidades de Producción Animal presenten parasitosis susceptibles a la IVM. Paraud et al., (2016) ha comprobado en Francia, que esto último no sólo puede modificar la efectividad de IVM, sino que además es posible la existencia 
de una resistencia cruzada, reportando una resistencia a las lactonas macrocíclicas en ovinos, ya que demostraron la primera resistencia múltiple de nematodos gastrointestinales contra la misma familia de antiparasitario; pudiendo ser un problema en el presente estudio, debido a que el $60 \%$ de las Unidades de Producción Animal evaluadas presentaron resistencia a la IVM, y la resistencia cruzada en Francia se observó en granjas sospechosas de resistencia.

Paradójicamente investigaciones realizadas en México por Rizo (2017), muestran la gran diversidad de plantas que presentan metabolitos capaces de inhibir el crecimiento y desarrollo de patógenos (Phytophthora ssp., Colletotrichum gloeosporioides, Moniliophthora roreri); una de estas plantas evaluadas ha sido el extracto de jengibre (Zingiber officinale). Estudios reportados por Lin et al. (2010), han comprobado el efecto del jengibre (Zingiber officinale) como desparasitante con respecto a la mortalidad y reducción de movilidad en las larvas de Anisakis simplex, una especie de nematodo gastrointestinal presente en mamíferos marinos, peces, crustáceos y humanos.

La efectividad del extracto de jengibre (Zingiber officinale) al 3\% para eliminar e inmovilizar las larvas de Oesophagostomum dentatum, es similar en una eficacia al $62 \%$ al de la IVM1\% a las $48 \mathrm{~h}$, comprobando en este estudio que la efectividad biológica del extracto de jengibre (Zingiber officinale) tiene un crecimiento con respecto a la curva de dosis respuesta de este extracto. En Japón se han realizado investigaciones sobre la actividad antihelmíntica de los compuestos aislados de la raíz del jengibre (Zingiber officinale), syhogaol, shogaol, y gingerol. Han comprobado que los compuestos anteriores matan y reducen la movilidad en larvas de Anisakis simplex, una especie de nematodo gastrointestinal presente en mamíferos marinos, peces, crustáceos y humanos entre las 24 y 72 h (Lin et al., 2010). A su vez, Acuña y Torres, (2010) en un estudio realizado de las propiedades medicinales del jengibre (Zingiber officinale), han reportado al Gingerol como el componente activo más estudiado por sus diversos efectos farmacológicos, entre los más destacados antinflamatorios y antihelmíntico.

De acuerdo con estos resultados, comprobando la efectividad de jengibre (Zingiber officinale), para eliminar e inmovilizar las larvas de Oesophagostomum dentatum, se ha hecho una parametrización adecuada al modelo matemático de Gompertz para estimar la dosis letal 50 (DL50) del extracto de jengibre; como se muestra en el cuadro 2, tomando los siguientes valores para la parametrización, de acuerdo con la aplicación Solver del programa Excel de Microsoft Office 2017 (Correa, 2004).

$$
\begin{array}{ccc}
y= & a^{*} \exp \left(-b^{*} \exp \left(-c^{*} t\right)\right) \\
a= & 62.40846543 \\
b= & 6.979583949 \\
c= & 2.910435786 \\
y=62.40846543^{*} \exp \left(-6.979583949^{*} \exp \left(-2.910435786{ }^{*} t\right)\right)
\end{array}
$$


Cuadro 2. Parametrización del modelo de Gompertz para determinar $\mathrm{DL}_{50}$ de jengibre

\begin{tabular}{ccccc}
\hline & \% Inclusión & \% EB & Gompertz & SC \\
\hline & 0.1 & 2.07868 & 0.338436046 & 3.028449019 \\
& 0.3 & 5.19798 & 3.382901301 & 3.294510684 \\
JENGIBRE & 0.5 & 11.4595 & 12.24378442 & 0.615102049 \\
(Zingiber officinale) & 0.7 & 24.2727 & 25.12103484 & 0.719671995 \\
& 0.9 & 36.0928 & 37.53441718 & 2.078260097 \\
& 1 & 45.3562 & 42.67651026 & 7.180737091 \\
& 2 & 60 & 61.13021894 & 1.277394859 \\
& 3 & 62.9262 & 62.33817928 & 0.345768365 \\
\hline
\end{tabular}

Los resultados ajustados al modelo paramétrico de Gompertz, han determinado que la DL50 del jengibre (Zingiber officinale) se obtiene con una inclusión del $1.1858 \%$ de este extracto; pudiendo así con este combatir un 50\% de las larvas de Oesophagostomum dentatum presentes en la raza de CPM evaluados. Diversos estudios realizados para estimar la DL50 han comprobado que el modelo matemático de Gompertz es uno de los más precisos, sin presentar gran margen de error, a comparación de otros modelos como lo son el polinómico, logístico y de regresión lineal (Molina y Melo, 2010).

Con respecto a los demás extractos vegetales evaluados, los resultados arrojados por el software Statgraphics 9.0 no han sido relevantes, ya que su porcentaje de efectividad no supera el $18 \%$.

\section{CONCLUSIONES}

La efectividad biológica del extracto de jengibre (Zingiber officinale) (3\%), es similar al de la ivermectina (1\%). en un $62 \%$ de las muestras evaluadas; por lo que se demuestra la efectividad del extracto para eliminar e inmovilizar las larvas de Oesophagostomum dentatum, con una dosis letal 50 es del $1.1858 \%$ estimada por el modelo matemático de Gompertz. Los extractos vegetales evaluados de orégano (Origanum vulgare), tomillo (Thymus) y hierbabuena (Mentha spicata), si bien, tuvieron reportes de ser evaluados anteriormente como antiparasitarios de nematodos gastrointestinales y reportes de antibacterianos, no presentan un porcentaje de efectividad biológica relevante (menor al $18 \%)$, contra las larvas de Oesophagostomum dentatum.

Es necesario continuar con las evaluaciones para poder comprobar in vivo los efectos del jengibre (Zingiber officinale), como antiparasitario en las producciones de cerdos de traspatio contra las larvas de Oesophagostomum dentatum, y así poder comparar su dosis y rentabilidad por kilogramo del animal, con respecto a la ivermectina. 


\section{AGRADECIMIENTOS}

Se agradece al Consejo Nacional de Ciencia y Tecnología (CONACyT), por el financiamiento otorgado para los estudios de maestría del segundo autor.

\section{LITERATURA CITADA}

ABBOTT WS. 1987. A method of computing effectiveness of an insecticide. Journal of the American Mosquito Control Association. 3(2) 302-303. ISSN: 8756-971X https://www.biodiversitylibrary.org/content/part/JAMCA/JAMCA_V03_N2_P302-303.pdf

ACUÑA O, Torres A. 2010. Aprovechamiento de las propiedades funcionales del jengibre (Zingiber officinale) en la elaboración de condimento en polvo, infusión filtrante y aromatizante para quema directa. Revista Politécnica. 29(1) 60-69 ISSN: 1390-0129 https://bibdigital.epn.edu.ec/bitstream/15000/4343/1/RP-No.29\%288\%29.pdf

AGUILAR LA, Florian CJ. 2016. Diagnóstico situacional de los parásitos gastroentéricos en la crianza artesanal de cerdos (Sus scrofa doméstica) de traspatio en la zona urbana del Municipio de Santo Tomas Departamento de Chontales. Tesis de licenciatura. Universidad Nacional Agraria. Pp. 5-6. https://repositorio.una.edu.ni/3421/

AGUILERA GS. 2012. Validación de seis fitofármacos: ajenjo, nogal, pasionaria, salvia, sábila y jengibre en pacientes adultos de diecinueve a setenta años de edad en Quito, junio-agosto de 2010. Tesis de licenciatura. Pontificia Universidad Católica del Ecuador. http://repositorio.puce.edu.ec/bitstream/handle/22000/5252/T-PUCE-

5478.pdf?sequence $=1$ \&isAllowed $=\mathrm{y}$

ALONSO DM, Arnaud OR, Becerra NR, Torres AJ, Rodríguez VR, Quiroz RR. 2015. Frequency of cattle farms with ivermectin resistant gastrointestinal nematodes in sheep in Veracruz, México. Veterinary Parasitology. 212(3-4), 439-443. ISSN: 0304-4017 https://www.sciencedirect.com/science/article/abs/pii/S0304401715003520

BARRERE F, Shakya KP, Menzies PI, Peregrine AS, Prichard RK. 2013. Assessment of benzimidazole resistance in Haemonchus contortus in sheep flocks in Ontario, Canada: Comparison of detection methods for drug resistence. Veterinary Parasitology. 198(1): 159-165. ISSN: 0304-4017.

https://www.sciencedirect.com/science/article/abs/pii/S0304401713004457

BOWMAN DD. 2013. Georgis' Parasitology for Veterinarians. $10^{\mathrm{a}}$ ed. Philadelphia, USA. $\begin{array}{llll}\text { Editorial Elsevier. } & \text { Pp. } & 496 . & \end{array}$ https://www.elsevier.com/books/georgis-parasitology-for-veterinarians/bowman/978-14557-4006-2 
CHÁVEZ RM, Reveles HR, Saldivar ES, Muñoz EJ, Morales VM, Moreno GM. 2006. Evaluación del albendazol, ivermectina y nitazoxanida en infeccion causada por Trichinella spiralis en modelo suino. Archivos Venezolanos de Farmacología y Terapéutica. 25:8-84. ISSN 0798-0264.

http://ve.scielo.org/scielo.php?pid=S0798-02642006000200008\&script=sci_arttext

COFFIN DL. 1952. Laboratorio clínico en medicina veterinaria. $3^{a}$ edición. Boston, Massachusetts. Pp.355

COLES GC, Jackson F, Pomroy WE, Prichard RK, Samson HG, Silvestre A, Taylor MA, Vercruysse J. 2006. The detection of anthelmintic resistance in nematodes of veterinary importance. Veterinary Parasitology. 136:167-185. ISSN: 0304-4017 https://www.sciencedirect.com/science/article/abs/pii/S0304401705005649

CORDERO CM, Rojo F, Martínez A, Sánchez M, Hernández S, Navarrete I. 2000. Parasitología Veterinaria. España: Ed. McGraw-Hill. Pp. 935 ISBN: 84-486-0236-6. https://dialnet.unirioja.es/servlet/libro?codigo $=489596$

CORREA HJ. 2004. RUMENAL: procedimiento para estimar los parámetros de cinética ruminal mediante la función Solver de Microsoft Excel®. Revista Colombiana de Ciencias Pecuarias. 17(3): 250-254. ISSN: 2256-2958. https://revistas.udea.edu.co/index.php/rccp/article/view/323947/20781127

COSIALLS IL, Solanas PA, Núñez PM, Jiménez FM, Miralles PD, Serra DG. 2000. Estadística aplicada con SPSS y Statgraphics. España. Edicions Universitat Barcelona. $\quad P p . \quad 119 . \quad$ ISBN: https://books.google.com.mx/books?hl=es\&lr=\&id=odNxBeRLXUEC\&oi=fnd\&pg=PA5\&d $\mathrm{q}=$ Statgraphics $+9.0 \&$ ots $=5 \mathrm{hbalg} 50 \mathrm{jt} \& \mathrm{sig}=\mathrm{U} 74 \mathrm{bUpOfwfagLOIGifWiQ2DFQxc \& redir}$ esc $=$ $\mathrm{y} \# \mathrm{v}=$ onepage $\& \mathrm{q}=$ Statgraphics $\% 209.0 \& \mathrm{f}=$ false

CRINGOLI G, Rinaldi L, Veneziano V, Capelli G, Scala A. 2004. The influence of flotation solution, simple dilution and the choice of McMaster slide area (volume) on the reliability of the McMaster technique in estimating the faecal egg counts of gastrointestinal strongyles and Dicrocoelium dendriticum in sheep. Veterinary Parasitology. 123:121-131. ISSN: 0304-4017 https://www.sciencedirect.com/science/article/abs/pii/S0304401704002377

DÁVILA JG, González SI, Báez OL, Gaona, ÁE, Zaragoza SE. 2017. Optimización de la destilación de Origanum Vulgare $L$, con efecto antifúngico en Moniliophthora roreri. Espacio I+D, Innovación Más Desarrollo. 6(14):138-151. ISSN: 2007-6703. https://doi.org/10.31644/IMASD.14.2017.a07 
ENCALADA ML, Tuyub SH, Ramírez VG, Mendoza GP, Aguilar ML, López AM. 2014. Phenotypic and genotypic characterization of Haemonchus spp. And other gastrintestinal nematodes resistant to benzimidazole in infected calves from the tropical regions of Campeche State, México. Veterinary Parasitology. 205: 246-254. ISSN: 0304-4017 https://www.sciencedirect.com/science/article/abs/pii/S0304401714003793

FRONTERA E, Pérez M, Alcaide M, Reina D. 2009. Patología parasitaria porcina: en imágenes. España: Ed. Servet. Pp. 268. ISBN: 84-92569-12-3. https://dialnet.unirioja.es/servlet/libro?codigo $=490758$

GAWEL A, Kotonski B, Madej JA, Mazurkiewicz M. 2003. Effect of silimarin on chicken and turkey broilers' rearing and the production indices of reproduction hen flocks. Medycyna Weterynaryjna. 59:517-520. ISSN: 0025-8628. https://eurekamag.com/research/034/804/034804936.php

GEURDEN T, Chartier C, Franke J, Di RA, Traversa D, Von SG, Demeler J, Vanimisetti HB, Bartram DJ, Denwood MJ. 2015. Anthelmintic resistance to ivermectin and moxidectin in gastrointestinal nematodes of cattle in Europe. Journal Parasitology Drugs $\begin{array}{llll}\text { Drug } & \text { Resist. } & \text { 5(3):163-171. ISSN: }\end{array}$ https://www.sciencedirect.com/science/article/pii/S2211320715300087

GUERRA C, Galán JA, Méndez J, Perea EM. 2008. Evaluación del efecto del extracto de orégano (Oreganum vulgare) sobre algunos parámetros productivos de cerdos destetados. Tumbaga. 1:6-29. ISSN Online: 2216-118x. https://dialnet.unirioja.es/servlet/articulo?codigo=3993531

HORVAT AJ, Petrovic M, Babic S, Pavlovic DM, Asperger D, Pelko S, Mance AD, Kastelan MM. 2012. Analysis, occurrence and fate of anthelmintics and their transformation products in the environment. Trends Analyt. Chem. 31:61-84. ISSN:01659936 https://www.sciencedirect.com/science/article/abs/pii/S0165993611002937

KAPLAN RM, Vidyashankar AN. 2012. An inconvenient truth: global worming and anthelmintic resistance. Veterinary Parasitology. 186:70-78. ISSN: 0304-4017 https://www.sciencedirect.com/science/article/abs/pii/S0304401711007801

LAGARTO PA, Tillán CJ, Cabrera GY. 1997. Toxicidad aguda oral del extracto fluido de Mentha spicata L. (hierbabuena). Revista Cubana de Plantas Medicinales. 2:6-8. ISSN: 1028-4796.

http://scielo.sld.cu/scielo.php?pid=S102847961997000200002\&script=sci_arttext\&tlng=p t 
LEMUS C, Ly CC. 2010. Estudio de sustentabilidad de cerdos mexicanos pelones y cuinos. La iniciativa Nayarita. Revista computarizada de producción porcina. 17 (2): 89 98. ISSN:1026-9053 http://www.iip.co.cu/RCPP/172/172_10artCLemus.pdf

LIN RJ, Chen CY, Lee JD, Lu CM, Chug LY, Yen CM. 2010. Larvicidal constituents of Zingiber officinale (ginder) against Anisakis simplex. Repositorio Institucional Universidad Médica de Kaohsiung. http://ir.lib.kmu.edu.tw/handle/310902000/18749

LOUIE K, Vlassoff A, Mackay AD. 2007. Gastrointestinal nematode parasites of sheep: A dynamic model for their effecton liveweight gain. International Journal Parasitol. 37:233241. ISSN: 0020-7519.

https://www.sciencedirect.com/science/article/abs/pii/S0020751906003511

MCARTHUR CL, Handel IG, Robinson A, Hodgkinson JE, Bronsvoort BM, Burden F, Kaplan RM, Matthews JB. 2015. Development of the larval migration inhibition test for comparative analysis of ivermectin sensitivity in vyathostomin populations. Veterinary Parasitology. 212: 292-298. ISSN: 0304-4017.

https://www.sciencedirect.com/science/article/abs/pii/S0304401715003003

MOLINA VF, Melo MS. 2010. Importance of the statistical method applied to calculate the $\mathrm{EC}_{50}$ and $\mathrm{EC}_{95}$ of some isothiocyanates evaluated against Rhizoctonia solani Kuhn. Agronomía Colombiana. 28(2):235-244. ISSN 0120-9965 http://www.scielo.org.co/scielo.php?script=sci_arttext\&pid=S0120-99652010000200013

MUNGUÍA XJ, Valenzuela MW, Leyva CJ, Morales PM, Figueroa CJ. 2013. Potencial de orégano como alternativa natural para controlar Haemonchus contortus en ovino de pelo. Revista Lationoameriacana de Recursos Naturales. 9 (1): 150-154. ISSN: 2594-0384. http://revista.itson.edu.mx/index.php/rlrn/article/view/222/157

PARAUD C, Marcotty T, Lespine A, Sutra JF, Pors I, Devos I. 2016. Cross- resistance to moxidectin and ivermectin on a meat sheep farm in France. Veterinary Parasitology. 226.88-92. ISSN: 0304-4017. https://www.sciencedirect.com/science/article/abs/pii/S0304401716302527

QUIROZ RH, Figueroa CJ, Ibarra VF y López AM. 2011. Epidemiología de enfermedades parasitarias en animales domésticos. México. 1a edición. Compact disc. Pp. 643. ISBN:978-607-00-4015-3.

https://www.academia.edu/31033300/Epidemiologia_de_enfermedades_parasitarias_en Animales_Domesticos 
RAMOS PA, Hernández SR. 2018. Extractos comerciales de tomillo (Thymus vulgaris) y de algarrobo (Ceratonia siliqua) en la dieta de lechones destetados. Revista Científica. 8:25-38. ISSN: 2221-5921.

http://revistas.unprg.edu.pe/openjournal/index.php/revistacientifica/article/view/249

REBOLLAR RA, Rebollar RS, Gómez TG, Hernández MJ, González RF. 2016. Crecimiento y especialización regional del sector pecuario en México, 1994 a 2013. Revista Mexicana de Ciencias Pecuarias. 7(3): 391-403. ISSN: 2428-6698 http://www.scielo.org.mx/scielo.php?pid=S2007-11242016000300391\&script=sci_arttext

RENDAL VM, Rodríguez CM, Fernández MR, Sánchez IJ, Segura IR, Veiga BA, Andión NC. 2004. Efecto del almacenamiento en fase gaseosa sobre la viabilidad celular, la apoptosis y la actividad funcional en aortas de cerdo criopreservadas. Estudio preliminar. Angiología. 56:107-121. ISSN: 0003-3170.

https://www.sciencedirect.com/science/article/abs/pii/S0003317004748555

RIZO MM, Vuelta LD, Lorenzo GA. 2017. Agricultura, desarrollo sostenible, medioambiente, saber campesino y universidad. Ciencia en su PC. (2):106-120. ISSN: 1027-2887.

https://www.redalyc.org/pdf/1813/181351615008.pdf

RODRÍGUEZ VR, Apanaskevich DA, Ojeda CM, Trinidad MI, Reyes NE, Esteve GM, de León AP. 2016. Ticks collected from humans, domestic animals, and wildlife in Yucatan, Mexico. Veterinary Parasitology. 215:106-113. ISSN: 0304-4017. https://www.sciencedirect.com/science/article/abs/pii/S0304401715300777

SALEM AZ, Salem MZ, El-Adawy MM, Robinson PH. 2006. Nutritive evaluations of some browse tree foliages during the dry season: secondary compounds, feed intake and in vivo digestibility in sheep and goats. Animal Feed Science and Technology. 127:251-267. ISSN: 377-8401. https://doi.org/10.1016/j.anifeedsci.2005.09.005

TAYLOR MA, Learmount J, Lunn E, Morgan C, Craign BH. 2009. Multiple resistence to anthelmintics in sheep and comparison of methods used for their detection. Small Ruminant Research. 86:67-70. ISSN: 0921-4488. https://doi.org/10.1016/j.smallrumres.2009.09.020

VAN WJ, Cabaret J, Michael LM. 2004. Morphological identification of nematode larvae of small ruminants and cattle simplified. Veterinary parasitology. 119:277-306. ISSN: 0304-4017. https://www.sciencedirect.com/science/article/abs/pii/S0304401703004734 